\title{
Slipped Capital Femoral Epiphysis as a Presentation of Underlying Metabolic Disorders: Pseudohypoparathyroidism and Juvenile Hypothyroidism
}

\author{
Rainel Zelaya ${ }^{1}$, Anthony Zarka ${ }^{2}$, Douglas Byerly ${ }^{3}$ \\ 1. Radiology, Brooke Army Medical Center, Fort Sam Houston, USA 2. Pediatric Radiology, Baylor College of \\ Medicine/Children's Hospital of San Antonio, San Antonio, USA 3. Department of Radiology, Wilford Hall Ambulatory \\ Medical Center, San Antonio, USA
}

Corresponding author: Rainel Zelaya, rainelzelaya@gmail.com

\begin{abstract}
Slipped capital femoral epiphysis (SCFE) is an abnormality of the proximal femoral physis typically occurring in adolescents and most commonly associated with obesity, although its exact etiology is unknown. In addition to obesity, other associations and predisposing factors proposed in the literature include trauma, vascular injury or compromise, and immunologic, genetic, and metabolic conditions. While not common, metabolic conditions are known to predispose to SCFE and it is essential for radiologists to recognize SCFE as a possible initial presentation of an underlying metabolic disorder. Understanding imaging findings and identification of atypical presentations of SCFE by radiologists can assist clinicians in guiding workup and lead to expedited treatment to prevent worsening outcomes associated with developmental delay.
\end{abstract}

Review began 03/01/2021 Review ended 03/07/2021 Published 03/09/2021

๑) Copyright 2021

Zelaya et al. This is an open access article distributed under the terms of the Creative Commons Attribution License CC-BY 4.0., which permits unrestricted use, distribution, and reproduction in any medium, provided the original author and source are credited.
Categories: Pediatrics, Radiology, Orthopedics

Keywords: scfe, pseudohypoparathyroidism, hypothyroidism, klein, salter-harris, greulich, pyle

\section{Introduction}

Slipped capital femoral epiphysis (SCFE) is a Salter-Harris type 1 injury involving the proximal femoral physis which results in eventual slippage of the proximal femoral epiphyses in relation to the metaphysis. The exact etiology of SCFE is disputable. However, both biomechanical and biochemical changes that occur at puberty are generally accepted etiologies. Age of presentation is affected by gender, with males typically presenting between the ages of 10-17 and females presenting slightly earlier between the ages of 8 and 15 [1]. Metabolic imbalances related to the thyroid, parathyroid, and pituitary glands such as hypothyroidism, pseudohypoparathyroidism, and hyperparathyroidism are known to be associated with SCFE [2]. In this case report, we present two patients presenting with imaging findings consistent with SCFE at the extremes of age than are typically seen with this condition, attributed to hypothyroidism and pseudohypoparathyroidism.

\section{Case Presentation}

\section{Case 1}

A 19-year-old male basic military trainee reported chronic, intermittent bilateral hip and groin pain since childhood, which acutely worsened over the past two weeks related to increased physical training. He reported mild discomfort associated with long periods of walking which increased with running, left greater than right. Pain decreased but persisted at rest. The patient's pain resulted in walking with a limp. Physical examination was notable for joint pain in both hips with active and passive motion, which worsened with weightbearing. The patient appeared younger than his stated age. Bilateral hip radiographs were obtained with frog leg views (Figure 1). Findings were concerning for left SCFE, possible right SCFE, and abnormal delayed skeletal maturation without significant closure of the femoral physes or apophyses. 


\section{Cureus}
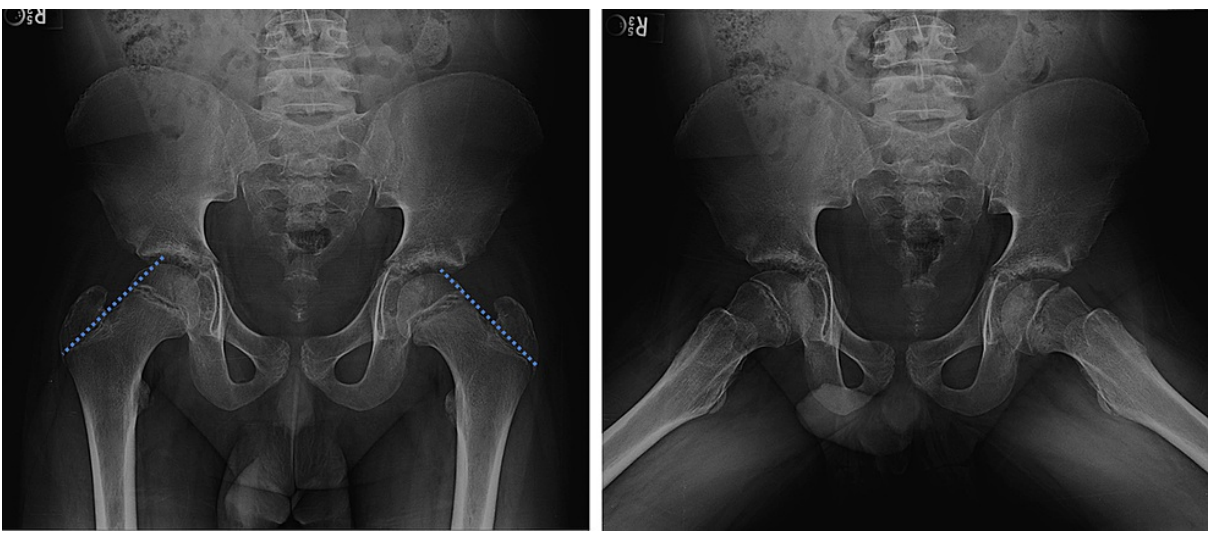

FIGURE 1: Plain radiographic AP (left image) and frog leg (right image) views of the bilateral hips. Suspected left SCFE in a 19-year-old male presenting with hip pain. AP view demonstrates lines of Klein (blue dotted lines). The left line does not intersect with the femoral epiphysis and passes above the left femoral head, supporting the diagnosis. There is slight intersection of the right femoral head by the line of Klein. Frog leg views demonstrate the posteromedial translation of the left proximal femoral epiphysis in relation to the metaphysis. The left femoral physis appears abnormally widened and irregular on both images. Note that the visualized osseous structures demonstrate abnormal delayed skeletal maturation, with no significant closure of the femoral physes or apophyses, inconsistent with reported age.

AP, anteroposterior; SCFE, slipped capital femoral epiphysis

The radiologist recommended orthopedic referral and obtaining a bone age assessment to estimate the amount of delay in skeletal maturation. He was subsequently admitted by the orthopedic department for treatment with bilateral surgical pinning. Magnetic resonance imaging (MRI) of the bilateral hips was ordered prior to surgery, which further supported findings consistent with left SCFE (Figure 2).
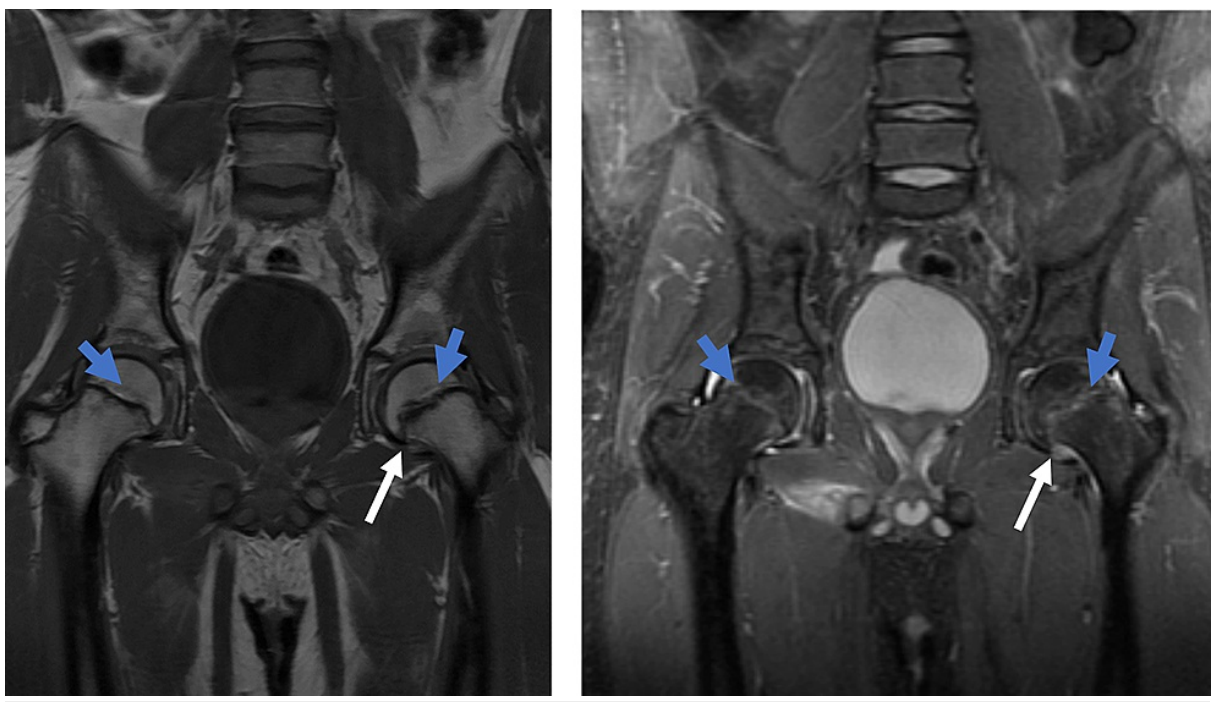

FIGURE 2: MRI of the bilateral hips. Coronal T1 (left image) and coronal STIR (right image) images of the bilateral hips demonstrate left greater than right periphyseal edema (blue arrows) characterized by mild STIR hyperintense and mild T1 hypointense signal with slip of the left femoral epiphysis (white arrows). Although there is some periphyseal 


\section{Cureus}

edema of the right femur, epiphyseal slippage is not evident. Open physes are again seen, representing delayed closure in this 19-year-old patient.

MRI, magnetic resonance imaging; STIR, short TI inversion recovery

Subsequent clinical follow-up initiated by his primary provider and the referred endocrinologist were notable for an elevated thyroid-stimulating hormone above $160 \mathrm{mcIU} / \mathrm{mL}$, a low level of free thyroxine, and an elevated serum thyroperoxidase antibody level of $765 \mathrm{IU} / \mathrm{mL}$, findings consistent with hypothyroidism likely secondary to Hashimoto thyroiditis. Further history obtained from the endocrinologist revealed symptoms consistent with hypothyroidism: fatigue, constipation, increased sleep, and problems with losing weight but without cold intolerance. A bone age study demonstrated a markedly delayed bone age, consistent with Greulich and Pyle male standard for 13 years and 6 months, incongruent with the patient's chronologic age of 19 years and 2 months (Figure 3).

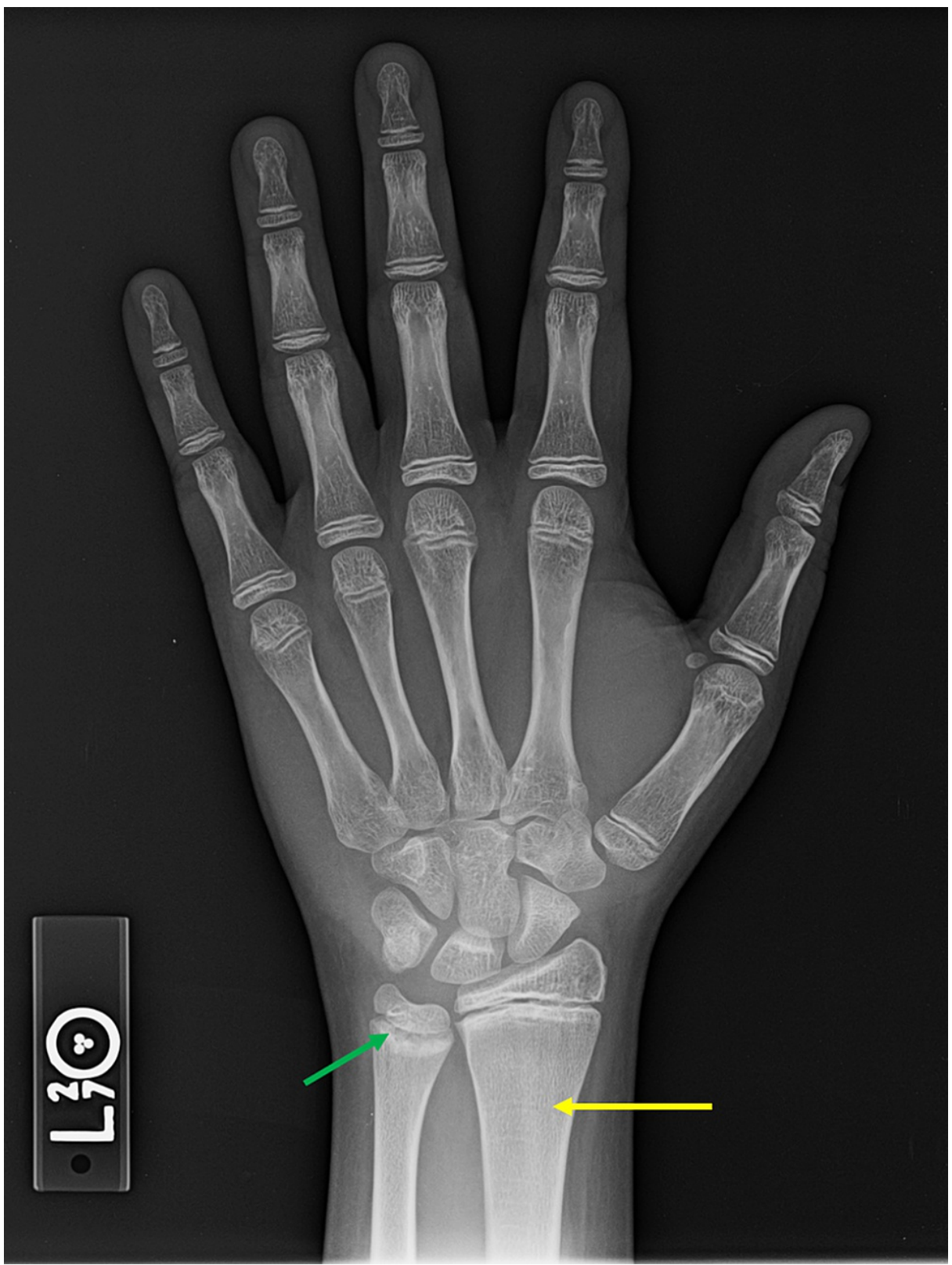

FIGURE 3: Bone age radiographs of the left hand and wrist. The patient most closely resembles the Greulich and Pyle male standard for 13 years and 6 months (162 months). The patient's chronologic age during time of this study was 19 years and 2 months ( 230 months). Note the 


\section{Cureus}

multiple growth recovery lines within the distal radial metadiaphysis (yellow arrow). There is relative increased density and mild cupping of the distal ulnar metaphysis (green arrow).

Following surgical pinning the patient was started on oral levothyroxine which was subsequently titrated as needed and demonstrated marked clinical improvement of his previous symptoms associated with hypothyroidism and with resolution of hip pain. Initial follow-up bone age studies demonstrated mild progression of physeal closure (not shown).

\section{Case 2}

A six-year-old female presented to her pediatrician with her father due to limping for two weeks. The child denied hip pain at the clinic visit but reported hip pain when sitting on the floor with her legs crossed for long periods of time. Physical examination of the hips was unremarkable, except for the inability to duck walk, and losing balance frequently with no pain report. Bilateral hip radiographs were obtained with frog leg views, demonstrating findings consistent with right SCFE, with possible left SCFE (Figure 4). Additionally noted were osseous findings consistent with an underlying metabolic or endocrine disease.
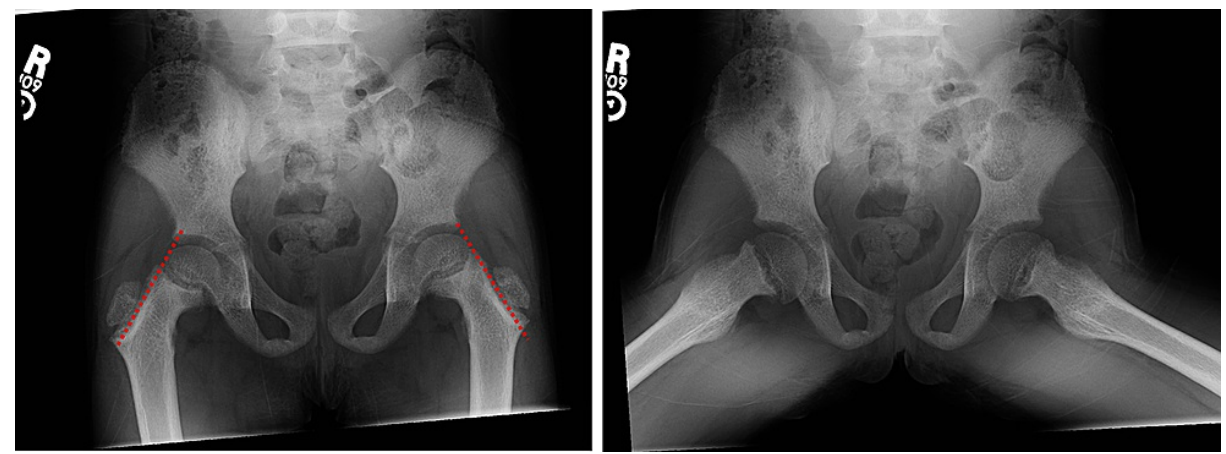

FIGURE 4: Plain radiographic AP (left image) and frog leg (right image) views of the bilateral hips. Six-year-old female presenting with limping. AP view demonstrates lines of Klein (red dotted lines) positioned above the femoral heads without intersection of the epiphysis. Frog leg views demonstrate the posteromedial translation of the proximal femoral epiphysis in relation to the metaphysis. Note the abnormal bone with coarsened trabecula, osteopenia, irregular widening of all the growth plates, and irregularity to the peripheral margins of the iliac crests. These findings were concerning for an underlying metabolic bone disease or endocrinopathy.

AP, anteroposterior

The patient was referred to orthopedics and placed on non-weightbearing status, with eventual workup for bilateral surgical pinning. An MRI of the bilateral hips obtained prior to surgery demonstrated mild bilateral, right greater than left, SCFE with redemonstrated findings supporting metabolic disease (Figure 5). 


\section{Cureus}
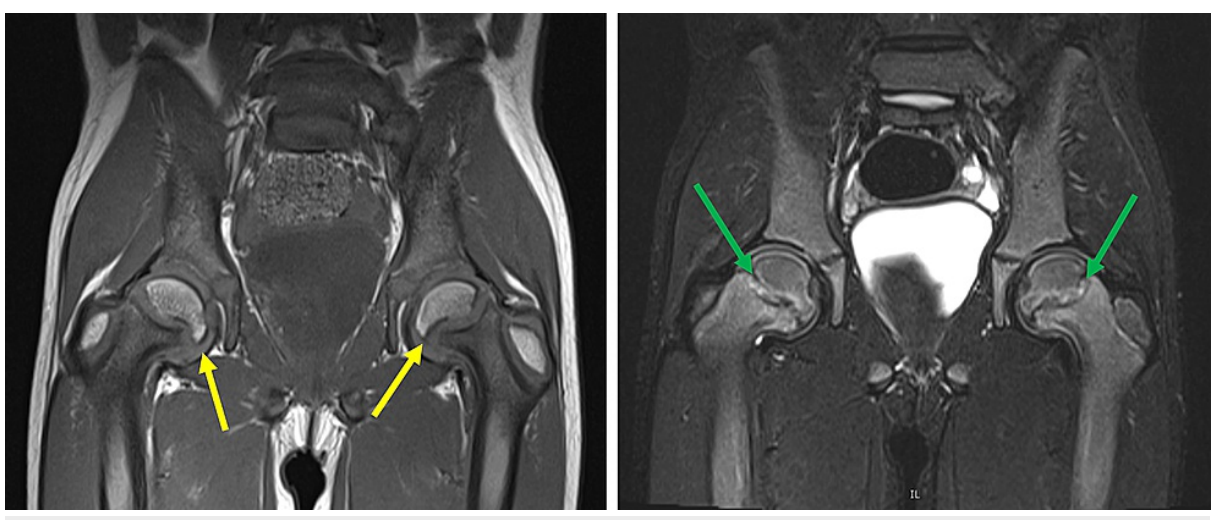

FIGURE 5: MRI of the bilateral hips. Coronal T1 (left image) and coronal STIR (right image) images of the bilateral hips redemonstrate bilateral right greater than left SCFE (yellow arrows). Accompanying moderate edema is noted along the metaphyseal and epiphyseal margins of the proximal femoral physes. Note bilateral coxa vara and irregular appearance of both proximal femoral metaphyses with mild widening and irregularity of the physes (green arrows), concerning for an underlying metabolic bone disease.

MRI, magnetic resonance imaging; STIR, short TI inversion recovery; SCFE, slipped capital femoral epiphysis

She was additionally referred to a pediatric endocrinologist after lab findings concerning for pseudohypoparathyroidism, with an elevated parathyroid hormone (PTH) at $607 \mathrm{pg} / \mathrm{mL}$, a mildly decreased serum calcium at $8.6 \mathrm{mg} / \mathrm{dL}$, and an elevated serum phosphate at $6.2 \mathrm{mg} / \mathrm{dL}$. Additional testing revealed metabolic PTH resistance. The clinical presentation was consistent with a de novo case of pseudohypoparathyroidism type $1 \mathrm{~b}$, which is characterized principally by $\mathrm{PTH}$ resistance. Calcium/calcitriol treatment was started, and the family was referred for genetic counseling. The patient had a normal postoperative course in the following months but was lost to follow-up afterward.

\section{Discussion}

Pediatric patients with hip pain or limping with subsequent radiographs should be evaluated for SCFE, and the remainder of the visualized skeletal structures must also be evaluated for additional abnormalities, as were present in both cases of patients presenting outside the typical age range of SCFE. Additional evaluation of remaining bone structures in addition to recognizing SCFE led to the workup of underlying endocrine disorders (hypothyroidism and pseudohypoparathyroidism type 1b), which may both predispose patients to develop SCFE [3-5].

Radiographic evaluation of pediatric patients with hip pain should include lines of Klein, a line along the superior border of the femoral neck which should cross the lateral part of the superior femoral epiphysis on an anteroposterior radiograph [6] (Figure 6). SCFE is diagnosed if the line does not cross the epiphysis and passes above the femoral head, as was present in both cases [7]. Other radiographic findings supporting the diagnosis depend on the presentation. Pre-slip images may demonstrate a widened physis along with irregularity and haziness of the physeal margins, and subsequently, a posteromedial acute slip which may be better visualized on the frog leg view [1]. 


\section{Cureus}

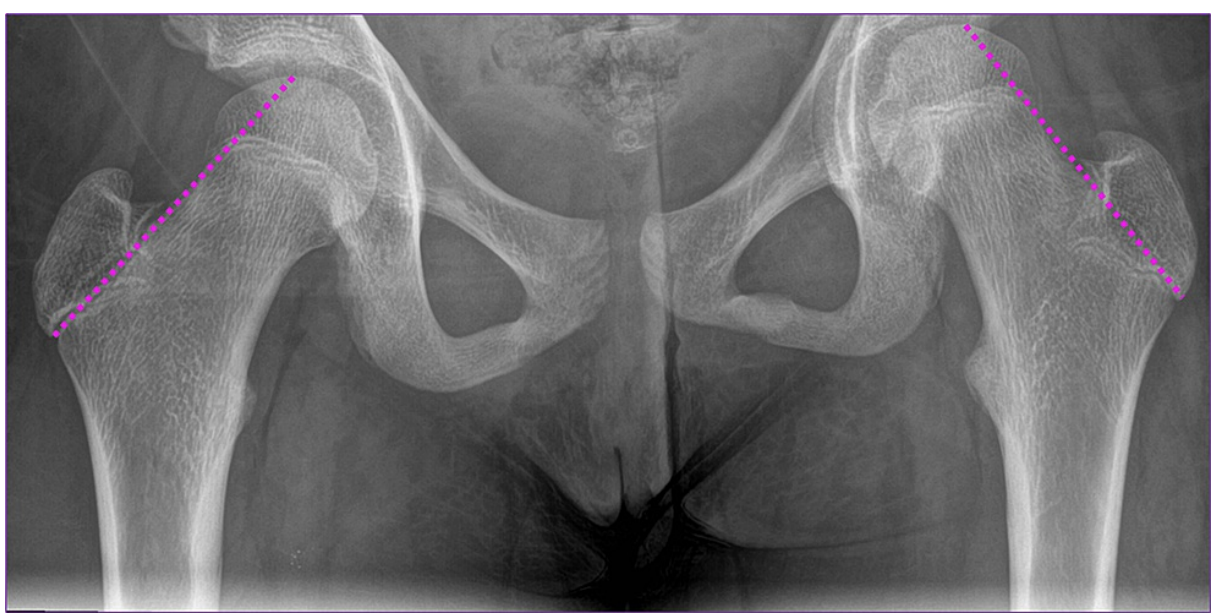

FIGURE 6: Normal AP hip radiograph of a 12-year-old female. Demonstrated are normal lines of Klein (pink dotted lines) drawn appropriately about the lateral proximal femurs. Note how both Klein lines intersect the femoral epiphyses.

AP, anteroposterior

Radiographs are the primary imaging modality for assessment of pediatric hip pain and SCFE, and recognition of expected findings of SCFE is essential for radiologists. Ultrasound findings are not specific and may demonstrate a femoroacetabular joint effusion, and possibly show malalignment between the epiphysis and metaphysis of the proximal femur. Although CT demonstrates the typical

epiphyseal/metaphyseal malalignment, ionizing radiation limits its use in pediatric patients and is therefore not recommended. MRI is helpful in the acute stage, typically demonstrating the malalignment in addition to increased T2 signal and corresponding low T1 signal, representing marrow edema surrounding the epiphyseal border [8]. MRI findings may also include an associated hip joint effusion as well as document preservation of blood flow to the femoral epiphysis if contrast is administered.

As both cases demonstrated, metabolic disturbances may predispose to SCFE, and examining osseous structures in patients with SCFE for signs of metabolic abnormalities or inconsistencies with patient age may be the first step in directing the appropriate workup to reverse the underlying cause. Several findings may suggest metabolic disorders, and pseudohypoparathyroidism shares many of the same skeletal features seen with other disorders such as Rickets, hyperparathyroidism, and osteomalacia [9]. These include metaphyseal irregularities, widening of the growth plates, osteopenia with decreased cortical and trabecular bone, coxa vara, and SCFE, most of which were seen in our second case.

Skeletal findings associated with congenital hypothyroidism are well documented in the literature; however, skeletal findings of juvenile hypothyroidism are less defined due to its scarcity. The most common cause of hypothyroidism is Hashimoto thyroiditis, as seen in our first case. Documented findings in the literature have included failed epiphyseal closure consistent with delayed bone age, dwarfism, and thickened bands at the metaphyseal ends, also known as growth recovery lines [10,11]. Other than dwarfism, the remainder of these findings were noted in our first case. If delayed bone age is suspected, dedicated bone age studies may be used to confirm the diagnosis [12,13].

Treatment of SCFE typically involves surgical pinning without reduction of the malalignment as this could compromise blood flow to the femoral epiphysis [14,15]. Lack of treatment may result in significant morbidity, including complications such as osteoarthrosis, avascular necrosis, chondrolysis, and limb length discrepancy $[16,17]$. Correction of the underlying metabolic disturbances is generally expediently started upon discovery to prevent further complications related to developmental delay [18,19].

\section{Conclusions}

Diagnosis of SCFE is crucial in the imaging evaluation of pediatric patients with hip pain or limping and may prevent significant morbidity directly related to SCFE, while also potentially unmasking an underlying metabolic disorder such as hypothyroidism or pseudohypoparathyroidism, as demonstrated by our two case presentations of patients presenting outside the age range that is typically seen with SCFE. It is also equally important for radiologists to carefully inspect the remaining visualized skeletal structures and consider bone age studies to substantiate the diagnosis of a metabolic derangement. 


\section{Additional Information \\ Disclosures}

Human subjects: Consent was obtained or waived by all participants in this study. Conflicts of interest: In compliance with the ICMJE uniform disclosure form, all authors declare the following: Payment/services info: All authors have declared that no financial support was received from any organization for the submitted work. Financial relationships: All authors have declared that they have no financial relationships at present or within the previous three years with any organizations that might have an interest in the submitted work. Other relationships: All authors have declared that there are no other relationships or activities that could appear to have influenced the submitted work.

\section{References}

1. Boles CA, El-Khoury GY: Slipped capital femoral epiphysis. Radiographics. 1997, 17:809-23. 10.1148/radiographics.17.4.9225384

2. Witbreuk M, van Kemenade FJ, van der Sluijs JA, Jansma EP, Rotteveel J, van Royen BJ: Slipped capital femoral epiphysis and its association with endocrine, metabolic and chronic diseases: a systematic review of the literature. J Child Orthop. 2013, 7:213-23. 10.1007/s11832-013-0493-8

3. Kadowaki S, Hori T, Matsumoto H, et al.: Prepubertal onset of slipped capital femoral epiphysis associated with hypothyroidism: a case report and literature review. BMC Endocr Disord. 2017, 17:59. 10.1186/s12902017-0210-6

4. Agarwal C, Seigle R, Agarwal S, Bilezikian JP, Hyman JE, Oberfield SE: Pseudohypoparathyroidism: a rare cause of bilateral slipped capital femoral epiphysis. J Pediatr. 2006, 149:406-8. 10.1016/j.jpeds.2006.04.057

5. Somasundaram KR, Sankararaman S, Siddiqui A, Zadeh H: Pseudohypoparathyroidism as a rare cause of bilateral slipped capital femoral epiphysis. Indian J Orthop. 2012, 46:705-9. 10.4103/0019-5413.104223

6. Klein A, Joplin RJ, Reidy JA, Hanelin J: Roentgenographic features of slipped capital femoral epiphysis . Am J Roentgenol Radium Ther. 1951, 66:361-74.

7. Georgiadis AG, Zaltz I: Slipped capital femoral epiphysis: how to evaluate with a review and update of treatment. Pediatr Clin North Am. 2014, 61:1119-35. 10.1016/j.pcl.2014.08.001

8. Tins B, Cassar-Pullicino V, McCall I: The role of pre-treatment MRI in established cases of slipped capital femoral epiphysis. Eur J Radiol. 2009, 70:570-8. 10.1016/j.ejrad.2008.02.049

9. Burnstein MI, Kottamasu SR, Pettifor JM, Sochett E, Ellis BI, Frame B: Metabolic bone disease in pseudohypoparathyroidism: radiologic features. Radiology. 1985, 155:351-6. 10.1148/radiology.155.2.3983385

10. Gutch M, Philip R, Philip R, Toms A, Saran S, Gupta KK: Skeletal manifestations of juvenile hypothyroidism and the impact of treatment on skeletal system. Indian J Endocrinol Metab. 2013, 17:181-3. 10.4103/22308210.119565

11. Edwards AM, Gray GC: Observations on juvenile hypothyroidism in native races of Northern Canada . Can Med Assoc J. 1961, 84:1116-24.

12. Greulich WW, Pyle SI: Radiographic atlas of skeletal development of the hand and wrist . Stanford University Press, Stanford, California; 1959.

13. Satoh M: Bone age: assessment methods and clinical applications . Clin Pediatr Endocrinol. 2015, 24:143-52. 10.1297/cpe.24.143

14. Hägglund G: Pinning the slipped and contralateral hips in the treatment of slipped capital femoral epiphysis. J Child Orthop. 2017, 11:110-3. 10.1302/1863-2548-11-170022

15. Seller K, Wild A, Westhoff B, Raab P, Krauspe R: Clinical outcome after transfixation of the epiphysis with Kirschner wires in unstable slipped capital femoral epiphysis. Int Orthop. 2006, 30:342-7. 10.1007/s00264006-0110-2

16. Carney BT, Weinstein SL: Natural history of untreated chronic slipped capital femoral epiphysis . Clin Orthop Relat Res. 1996, 43:7.

17. Kennedy JG, Hresko MT, Kasser JR, Shrock KB, Zurakowski D, Waters PM, Millis MB: Osteonecrosis of the femoral head associated with slipped capital femoral epiphysis. J Pediatr Orthop. 2001, 21:189-93.

18. Mantovani G, Bastepe M, Monk D, et al.: Diagnosis and management of pseudohypoparathyroidism and related disorders: first international Consensus Statement. Nat Rev Endocrinol. 2018, 14:476-500. 10.1038/s41574-018-0042-0

19. Garber JR, Cobin RH, Gharib H, et al.: Clinical practice guidelines for hypothyroidism in adults: cosponsored by the American Association of Clinical Endocrinologists and the American Thyroid Association. Endocr Pract. 2012, 18:988-1028. 10.4158/EP12280.GL 